\title{
Anomalous Origin of Left Main Coronary Artery from the Right Coronary Artery: Echocardiographic Diagnosis
}

\author{
Luca Felice Cerrito', Valentina Battisti ${ }^{1}$, Roberto Malagò², \\ Giancarlo Mansueto ${ }^{2}$, Andrea Rossi ${ }^{1}$ \\ 'Section of Cardiology, Department of Medicine, University of Verona, Verona, Italy \\ ${ }^{2}$ Department of Radiology, G.B. Rossi Policlinic, University of Verona, Verona, Italy
}

Received: June 6, 2019

Revised: July 3, 2019

Accepted: July 11, 2019

Corresponding author

Luca Felice Cerrito, MD

Section of Cardiology,

Verona University Hospital,

University of Verona,

Piazzale Stefani 1,

37126 Verona, Italy

Tel: 39-347-9907453

Fax: 39-045-8027307

E-mail: lucacerry90@gmail.com

\begin{abstract}
An anomalous origin of the coronary artery is rarely seen in clinical practice, and left coronary artery arising from the right coronary artery is an extremely rare anomalous coronary anomaly. Echocardiographic assessment of coronary arteries is an important component of routine cardiovascular evaluation, but detection of an anomalous coronary origin remains a challenge to operators. We report a case of a 42-year-old women presenting to the hospital with atypical chest pain for whom echocardiographic evaluation revealed a coronary artery anomaly.
\end{abstract}

Key words Coronary artery $\cdot$ Coronary vessels, anomalies $\cdot$ Tomography Echocardiography $\cdot$ Heart defect, congenital.

\section{INTRODUCTION}

Echocardiographic assessment of the coronary arteries is an important component of cardiovascular evaluation, but detection of a coronary artery anomaly (CAA) remains a challenge. CAAs are most often classified into abnormalities of origin, distribution, or termination and are found in $0.2-1.6 \%$ of those undergoing coronary angiography $[1,2]$. Anomalous origin of left coronary artery (LCA) is 6 to 8 time less common than that of right coronary artery (RCA). In this report, we present an anomalous origin of the left main coronary artery (LM) from the RCA diagnosed by routine transthoracic echocardiography (TTE).

\section{CASE REPORT}

A 42-year-old women with no cardiovascular risk factors was admitted to the internal medicine department for evaluation of atypical chest pain. There was no reported history of lightheadedness, dizziness, or syncope. Physical examination, biochemical values, and electrocardiography (ECG) were normal. The patient underwent a cycle stress test with no ischemic changes

(c) This is an Open Access article distributed under the terms of the Creative Commons Attribution Non-Commercial License (https://creativecommons.org/licenses/by$\mathrm{nc} / 4.0)$ which permits unrestricted non-commercial use, distribution, and reproduction in any medium, provided the original work is properly cited. on ECG and no angina. TTE revealed normal left ventricular systolic function with no segmental kinetic abnormalities and an anomalous coronary arising from an ectatic RCA (Fig. 1); furthermore, the origin of the LM was not detectable in the left cusp region. Subsequently, the patient underwent CT angiogram for assessment of coronary lesions, which disclosed an anomalous origin of the LCA from the ostial RCA. The LCA traveled to the left ventricle, anterior to the aorta and pulmonary artery, and distally branches in the circumflex and left anterior descending arteries (Fig. 2). No luminal stenosis was noted on CT angiogram. The heart surgeon of our hospital was consulted: because of no interarterial LM course, absence of intra-mural course, and no clear heart-related symptoms, the patient was excluded from surgery.

\section{DISCUSSION}

CAAs are generally silent and usually diagnosed accidentally during coronary angiography or autopsy [3]. An LM originating from the right sinus of Valsalva as a separate vessel or as a branch of a single coronary artery is an extremely rare anomaly, with an incidence up to $0.11 \%$, and represents a life-threatening form of congenital pathology [4]. Five types have been described according to relationships with the aorta and pulmonary artery 

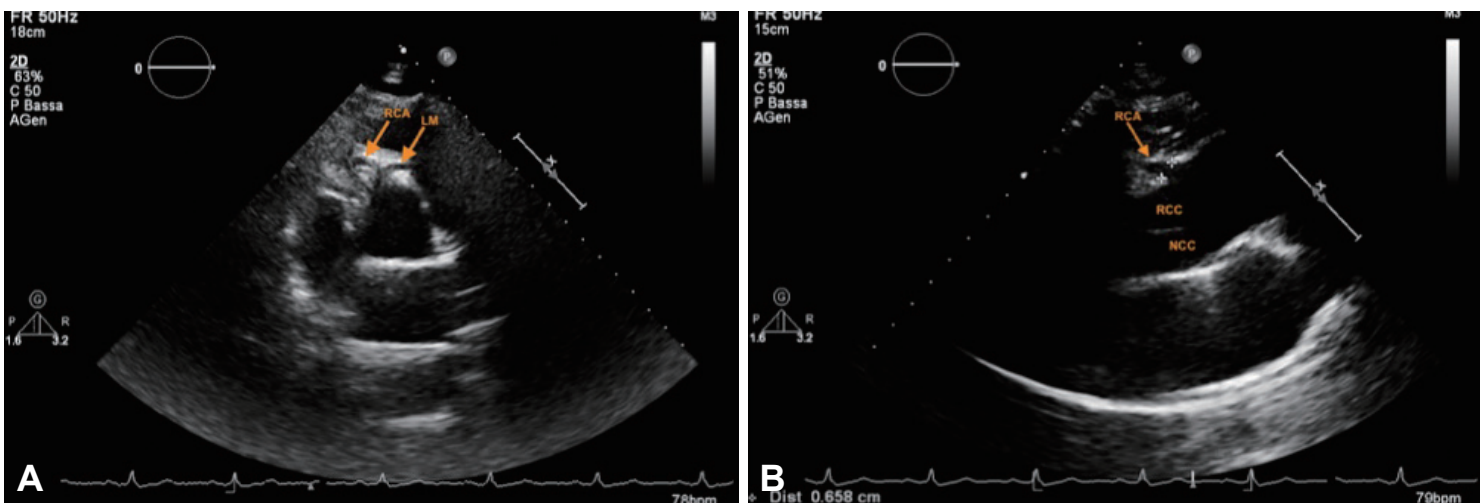

Fig. 1. Transthoracic echocardiography approach. A: Modified parasternal short axis demonstrating RCA origin from the RCC and LM origin from the RCA. B: Standard parasternal long axis showing an ectatic RCA takeoff from the RCC. RCA: right coronary artery, RCC: right coronary cusp, NCC: non coronary cusp, LM: left main coronary artery.
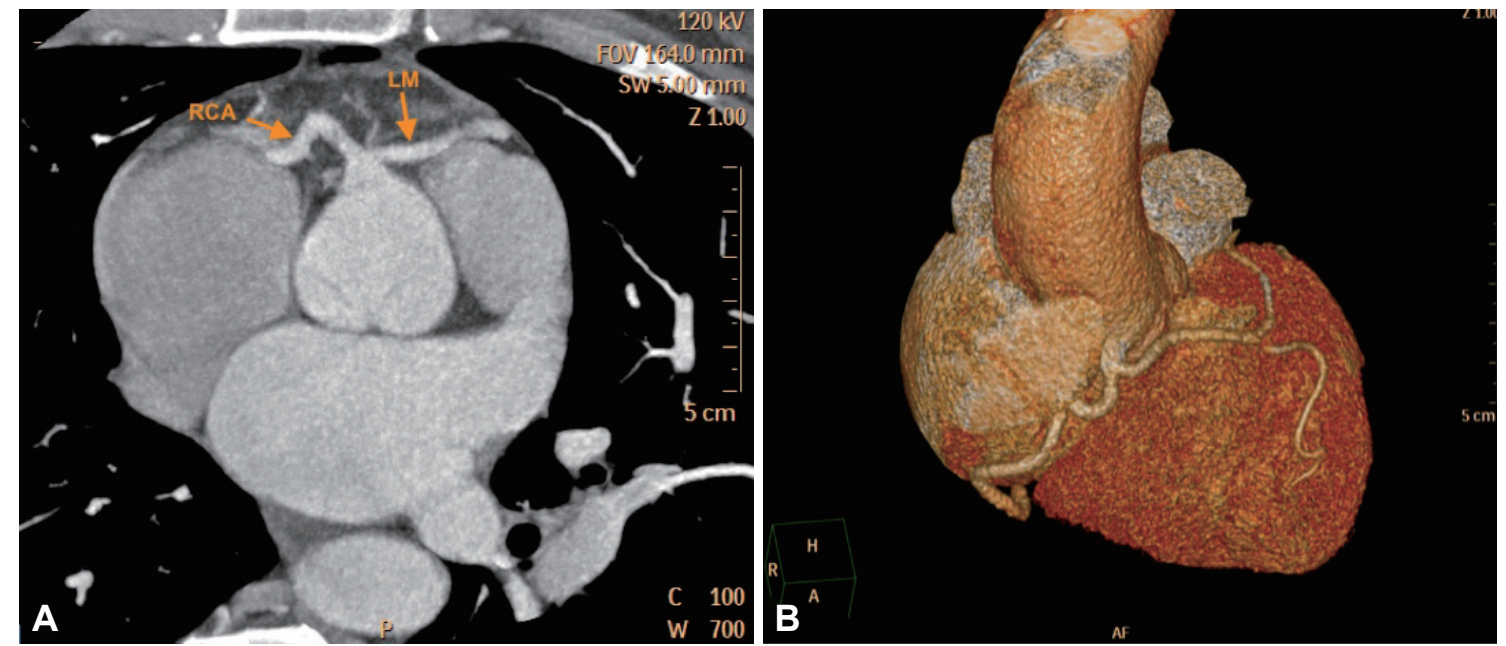

Fig. 2. Coronary CT approach. A: CT images of anomalous LM takeoff from the ostial RCA. B: Volume-rendered image showing the anomalous origin of the left coronary artery, as well as its course crossing the right ventricular outflow tract. CT: computed tomography, RCA: right coronary artery, LM: left main coronary artery.

Table 1. Variants of ectopic origin of left coronary artery from the right sinus of Valsalva

\begin{tabular}{|c|c|}
\hline $\begin{array}{l}\text { Coronary } \\
\text { artery variant }\end{array}$ & Description of vascular pathway \\
\hline Anterior & The LM trunk traverses the epicardium and crosses the right ventricular outflow tract before dividing into the LAD and LCX \\
\hline Septal & $\begin{array}{l}\text { The LM trunk produces a primary septal branch that travels intramuscularly before assuming an epicardial position } \\
\text { on the posterior aspect of the heart }\end{array}$ \\
\hline Combined & $\begin{array}{l}\text { The LAD travels anterior to PA, the septal branch travels intramuscularly toward the posterior aspect of the heart, } \\
\text { and the LCX travels posterior to the aorta }\end{array}$ \\
\hline Posterior & The LM travels posterior to the aorta before splitting into the LAD and LCX \\
\hline Between & The LM originates from the RCA and travels posteriorly between the aorta and PA prior to dividing into the LAD and LCX \\
\hline
\end{tabular}

The variants are listed in order of increasing severity from top to bottom [3]. LM: left main coronary artery, PA: pulmonary artery, LCX: left circumflex artery, LAD: left anterior descending coronary artery, RCA: right coronary artery

(Table 1) [3].

Precise images of anomalous anatomy are essential to appropriate management of the condition. Although coronary angiography is typically used, CT angiogram should be the method of choice to detect and classify CAA anatomy [5]. Despite the previously mentioned methods, TTE remains the first-line ex- amination due to its availability and non-invasive nature. Identification of the origins and proximal courses of the coronary arteries may often be achieved with TTE. The parasternal shortaxis view at the base of the heart is the best to determine both the relationship of the origin of the LM to the zone of apposition between the aortic valvular leaflets guarding the coronary 
aortic sinuses and its relationship to the right ventricular outflow tract. Due to extreme translation of the coronary arterial origins during the cardiac cycle, the excellent temporal resolution of echocardiography compared with that of other noninvasive techniques makes this the diagnostic technique of choice in most cases. Even though it is not common in daily practice, the origin of the coronary artery was identified by Labombarda et al. [6] in $91 \%$ of young adults, and more than half of the patients had undergone at least one TTE before the CAA was discovered. According to this data, CAA is underdiagnosed. In the present case, the coronary artery origin and course were well identifiable. This study was performed by operators experienced in analysis of coronary arteries. In conclusion, anomalous origin of the LM from the RCS is a rare condition. Coronary anatomy should be carefully analyzed with TTE; when CAA is suspected, CT angiogram should be performed.

\section{Conflicts of Interest}

The authors have no potential conflicts of interest to disclose.

\section{REFERENCES}

1. Cieslinski G, Rapprich B, Kober G. Coronary anomalies: incidence and importance. Clin Cardiol 1993;16:711-715.

2. Baltaxe HA, Wixson D. The incidence of congenital anomalies of the coronary arteries in the adult population. Radiology 1977;122:47-52.

3. Yamanaka O, Hobbs RE. Coronary artery anomalies in 126,595 patients undergoing coronary arteriography. Cathet Cardiovasc Diagn 1990;21:2840.

4. Bunce NH, Lorenz CH, Keegan J, Lesser J, Reyes EM, Firmin DN, et al. Coronary artery anomalies: assessment with free-breathing three-dimensional coronary MR angiography. Radiology 2003;227:201-208.

5. Akcay A, Tuncer C, Batyraliev T, Gokce M, Eryonucu B, Koroglu S, et al. Isolated single coronary artery: a series of 10 cases. Circ J 2008;72:12541258.

6. Labombarda F, Coutance G, Pellissier A, Mery-Alexandre C, Roule V, Maragnes $\mathrm{P}$, et al. Major congenital coronary artery anomalies in a paediatric and adult population: a prospective echocardiographic study. Eur Heart J Cardiovasc Imaging 2014;15:761-768. 